\title{
JD4 - Progress in Understanding the Physics of Ap and Related Stars
}

\section{Preface}

Chemically peculiar (CP) stars exhibit, simultaneously, a wide variety of physical phenomena, including diffusion, convection, magnetism, and pulsation. Thus, progress in the understanding of these objects requires the input of researchers from a variety of research fields within stellar astrophysics. The General Assembly of the IAU, in Rio de Janeiro, provided an excellent opportunity to discuss challenging new results faced in $\mathrm{CP}$ star research and improve the exchange of information and cooperation with experts of neighbouring scientific fields.

The Joint Discussion, entitled "Progress in understanding the physics of Ap and related stars", of 1.5 days in length, was organized by the Working Group on Ap and related stars. It comprised three sessions, each with a number of review talks along with short oral contributions. During the Joint Discussion a variety of important topics were discussed, such as the optimal exploitation of current and future instrument capabilities in the context of CP star research, the modelling of the complex atmospheres of peculiar stars and a look at $\mathrm{CP}$ and related phenomena in the context of Stellar Evolution. Reviews of the science highlights presented during each of the Joint Discussion's sessions are presented in these proceedings.

One important aspect of the scientific programme was the discussion that followed each session. During these sessions, the participants addressed questions such as "To what degree may complementary techniques contribute to the understanding of the CP phenomenon?", "Atomic data: what do we need as input? How confidently can we derive Global parameters of CP stars?" and "What is the key to the diversity of CP phenomena? The roles of magnetic fields, rotation, diffusion, birth environment, and binarity." . We are very grateful to Laurent Eyer, Glenn Wahlgren and Nikolai Piskunov for organizing these most fruitful discussion sessions. Similarly, we would like to thank all of those that contributed to the organization of this Joint Discussion, and all speakers and poster presenters that made the Joint Discussion possible.

Margarida S. Cunha, Chair SOC

December, 2009 